\title{
Desconfortos associados às cirurgias de extração dentária e construção de instrumento de medida (QCirDental). Parte I: impactos e consistência interna
}

\author{
Discomfort associated with dental extraction surgery \\ and development of a questionnaire (QCirDental). \\ Part I: Impacts and internal consistency
}

\author{
Marcelo Carlos Bortoluzzi ${ }^{1}$ \\ Luciana Dorochenko Martins ${ }^{1}$ \\ André Takahashi ${ }^{1}$ \\ Bianca Ribeiro ${ }^{1}$ \\ Ligiane Martins ${ }^{2}$ \\ Marcia Helena Baldani Pinto ${ }^{1}$
}

${ }^{1}$ Departamento de Odontologia, Setor de Ciências Biológicas e da Saúde, Universidade Estadual de Ponta Grossa. Praça Santos Andrade s/n, Centro. 84030-000 Ponta Grossa PR Brasil. mbortoluzzi@gmail.com ${ }^{2}$ Programa de Mestrado em Biociências e Saúde, Universidade do Oeste de Santa Catarina. Joaçaba SC Brasil.

\begin{abstract}
The scope of this study was to develop and validate a questionnaire (QCirDental) to measure the impacts associated with dental extraction surgery. The QCirDental questionnaire was developed in two steps; (1) question and item generation and selection, and (2) pretest of the questionnaire with evaluation of the its measurement properties (internal consistency and responsiveness). The sample was composed of 123 patients. None of the patients had any difficulty in understanding the QCirDental. The instrument was found to have excellent internal consistency with Cronbach's alpha reliability coefficient of 0.83. The principal component analysis (Kaiser-Meyer-Olkin Measure of Sampling Adequacy 0,72 and Bartlett's Test of Sphericity with $p<0.001$ ) showed six (6) dimensions explaining $67.5 \%$ of the variance. The QCirDental presented excellent internal consistency, being a questionnaire that is easy to read and understand with adequate semantic and content validity. More than $80 \%$ of the patients who underwent dental extraction reported some degree of discomfort within the perioperative period which highlights the necessity to assess the quality of care and impacts of dental extraction surgery.

Key words Oral surgery, Validation study, Questionnaires
\end{abstract}

Resumo O estudo objetivou criar um instrumento de medida que pudesse quantificar os desconfortos associados ao procedimento cirúrgico de pacientes submetidos a cirurgias dento-alveolares (QCirDental). O instrumento QCirDental foi desenvolvido em duas etapas iniciais: (1) geração e seleção das perguntas ou itens e, (2) teste do instrumento com avaliação das propriedades de medida (consistência interna e responsividade). A amostra foi composta por 123 pacientes. Nenhum deles apresentou qualquer dificuldade em entender o QCirDental. O instrumento mostrou excelente consistência interna com coeficiente alfa de Cronbach de 0,83. A análise de componentes principais (Kaiser-Meyer-Olkin Measure of Sampling Adequacy 0,72 e Bartlett's Test of Sphericity com $p<0,001)$ mostrou seis (6) dimensões do instrumento que explicam $67,5 \%$ da variância. $O$ QCirDental possui excelente consistência interna, sendo um instrumento de fácil leitura e interpretação compativel com uma adequada validade semântica e de conteúdo. Mais de $80 \%$ dos pacientes submetidos a extrações dentárias de rotina odontológica apresentam algum desconforto ou incomodo no período transoperatório o que ressalta a necessidade de utilização de um instrumento para avaliação de cuidados.

Palavras-chave Cirurgia bucal, Estudo de validação, Questionários 


\section{Introdução}

Numerosos instrumentos de medida têm sido desenvolvidos nos últimos anos com o objetivo de quantificar os impactos da condição bucal sobre diversos aspectos da vida do indivíduo tais como o bem estar social, psicológico e a qualidade de vida, além de procurar medir características relacionadas à função e aos sintomas.

Diferentes problemas ou condições bucais podem refletir de forma distinta sobre os indivíduos. Dessa forma, questionários buscando verificar os impactos das condições ou doenças especificas têm sido cada vez mais descritos por atribuírem uma melhor capacidade discriminatória aos problemas pontuais e, portanto, permitirem uma leitura mais fidedigna da condição e suas repercussões sobre o indivíduo ${ }^{1-8}$. Todavia, a maioria dos estudos relacionados à cirurgia bucal tende a quantificar e descrever os impactos, as consequências, os resultados e os desconfortos das terapias empregadas somente no pós-procedimento e, quando o transoperatório é avaliado, em geral a dor é o único item de interesse? ${ }^{9}$.

Genericamente, o cirurgião-dentista realiza os procedimentos cirúrgicos e de extração dentária de rotina atento à técnica e, em geral, objetivando a reabilitação. Entretanto, normalmente demonstra estar pouco ciente dos impactos significantes e negativos para a qualidade de vida do paciente que a perda dentária traz ${ }^{10}$, tampouco, busca conhecer todos os incômodos que o procedimento em si evidencia. A qualidade do cuidado tem sido descrita como parte crucial do tratamento, apregoando-se a inclusão da perspectiva do paciente como parte fundamental na integralidade da terapia. Contudo, pouca atenção tem sido dada a essa visão no que tange ao processo de intervenção propriamente dito ${ }^{11}$. O único instrumento disponível na literatura para medir a qualidade do processo da intervenção em cirurgia bucal foi descrito recentemente por Reissmann et al. ${ }^{11}$, e este é composto por 16 questões, incluindo sete perguntas relacionadas à anestesia, cinco sobre pressões e vibração, e uma para barulhos, gosto, dor e tempo cirúrgico.

O objetivo do presente estudo foi de criar um novo instrumento de medida que pudesse quantificar os impactos negativos e os desconfortos associados ao procedimento cirúrgico no período trans e perioperatório imediato de pacientes submetidos a cirurgias dento-alveolares (QCirDental), gerando dessa forma, condições de reconhecer falhas e identificando indivíduos mais vulneráveis com o intuito de melhorar e orientar o conjunto total de ações preventivas e protetoras aos pacientes.

\section{Materiais e Métodos}

\section{Desenho do estudo}

Este estudo contou com a apreciação e a aprovação do Comitê de Ética em Pesquisa da Universidade Estadual de Ponta Grossa (UEPG) e caracteriza-se por ser observacional e complementado por desenvolvimento e validação de instrumento.

\section{Desenvolvimento do Instrumento}

(Validade de Face e Validade de Conteúdo)

Utilizando o método descrito anteriormen$\mathrm{te}^{1,2,8}, \mathrm{o}$ instrumento QCirDental (Quadro 1) foi desenvolvido em duas etapas iniciais: (1) geração e seleção das perguntas ou itens, (2) teste do instrumento com avaliação das propriedades de medida (consistência interna e responsividade) com a possibilidade de redução do número de itens ${ }^{1,2,8}$.

A primeira etapa envolveu a geração dos itens do questionário que poderiam refletir a sensação de desconforto por parte do paciente quanto ao procedimento cirúrgico a que seria submetido. Os itens elaborados foram derivados da revisão da literatura e de entrevistas com cirurgiões maxilo-faciais e pacientes. A revisão da literatura abrangeu publicações de pesquisas que incluíssem instrumentos validados com enfoque especial em desconfortos em cirurgia bucal de extração dental ou cirurgia dento-alveolar localizadas nas seguintes bases de dados: PubMed (Medline), Lilacs, SciELO e BBO. Nestas bases, foram realizadas buscas restritas aos últimos 15 anos (2000-2015), com os termos: "oral surgery", "patient complaints", "dental extraction", "development of questionnaire", "patient disconfort", "measure instrument" e "patient satisfaction". Para a geração de itens, foram ainda consultados cirurgiões maxilo-faciais, professores universitários pertencentes aos quadros funcionais de uma instituição pública e duas privadas, via email, o que resultou na contribuição e em comentários de 5 profissionais. Itens ainda foram obtidos a partir de uma amostra de conveniência de pacientes, valendo-se de entrevista não estruturada e informal após cirurgias dento-alveolares realizadas em uma clínica de cirurgia dentária de uma universidade privada, e consistiu basicamente da seguinte pergunta "Durante a sua cirurgia, o que mais lhe incomodou?" Do conjunto, foram lista- 
Quadro 1. Questionário de autopercepção de cirurgia bucal dento-alveolar (QCirDental).

Nome:

Tente classificar o que mais lhe INCOMODOU durante a cirurgia em sua boca, conforme a pergunta.

USE A ESCALA, e selecione um número que melhor corresponde como você se sentiu.

\begin{tabular}{|c|c|c|c|c|c|c|c|c|c|c|}
\hline $\begin{array}{c}0 \\
\text { Não incomodou nada ou a pergunta } \\
\text { não se aplica para minha cirurgia }\end{array}$ & 1 & 2 & 3 & 4 & 5 & 6 & 7 & \begin{tabular}{l|l}
8 & 9
\end{tabular} & \multicolumn{2}{|c|}{$\begin{array}{c}10 \\
\text { Incomodou muito/ } \\
\text { demais ou um absurdo }\end{array}$} \\
\hline & & & & & & & & & & Nota \\
\hline \multicolumn{9}{|l|}{ 1. Eu me senti nervoso durante a cirurgia } & \multirow{20}{*}{$\begin{array}{c}\text { E isso me } \\
\text { incomodou }\end{array}$} & 1. \\
\hline \multicolumn{9}{|c|}{ 2. Os comentários que os cirurgiões ou auxiliares fizeram durante a minha cirurgia } & & 2. \\
\hline \multicolumn{9}{|l|}{ 3. Os líquidos e sangue na minha boca } & & 3. \\
\hline \multicolumn{9}{|c|}{ 4. A impressão que eu tive dos machucados na minha boca } & & 4. \\
\hline \multicolumn{9}{|l|}{ 5. Eu tive medo da anestesia } & & 5. \\
\hline \multicolumn{9}{|l|}{ 6. A dor que eu senti durante a anestesia } & & 6. \\
\hline \multicolumn{9}{|l|}{ 7. A dor que eu senti durante a cirurgia } & & 7. \\
\hline \multicolumn{9}{|l|}{ 8. Os barulhos dos instrumentos } & & 8. \\
\hline \multicolumn{9}{|l|}{ 9. O tempo que a cirurgia levou } & & 9. \\
\hline \multicolumn{9}{|c|}{ 10. A falta de explicação do que estava acontecendo durante a cirurgia } & & 10. \\
\hline \multicolumn{9}{|c|}{ 11. A falta de delicadeza ou cuidado do cirurgião para comigo durante a cirurgia } & & 11. \\
\hline \multicolumn{9}{|c|}{ 12. Eu me senti indignado durante a cirurgia (por qualquer motivo relacionado) } & & 12. \\
\hline \multicolumn{9}{|c|}{ 13. A dificuldade do cirurgião para terminar a cirurgia } & & 13. \\
\hline \multicolumn{9}{|c|}{ 14. Durante a minha cirurgia, senti a minha privacidade invadida } & & 14. \\
\hline \multicolumn{9}{|l|}{ 15. O lugar, o ambiente } & & 15. \\
\hline \multicolumn{9}{|l|}{ 16. Os cheiros diferentes } & & 16. \\
\hline \multicolumn{9}{|c|}{ 17. Os materiais ou instrumentos que colocaram na minha boca } & & 17. \\
\hline \multicolumn{9}{|c|}{ 18. Eu me senti angustiado durante a cirurgia } & & 18. \\
\hline \multicolumn{9}{|c|}{ 19. A falta de explicações após terminar a cirurgia } & & 19. \\
\hline \multicolumn{9}{|c|}{ 20. A sensação de ter perdido meu(s) dente(s) } & & 20. \\
\hline
\end{tabular}

das questões consideradas relevantes e constantes no que tange às queixas frequentes percebidas na atividade clínica.

O conteúdo da primeira versão foi resubmetido aos cirurgiões maxilo-faciais (painel de especialistas) para análise qualitativa dos itens, pertinência, relevância, conteúdo e abrangência do instrumento. Em relação à validade aparente, os juízes consideraram os itens como claros e compreensíveis com concordância superior a $80 \%$. A análise semântica promoveu pequenos ajustes além da exclusão de redundâncias, porém, sem a necessidade de exclusão de assuntos listados como desconfortos.

O instrumento de avaliação da autopercepção da cirurgia bucal dento-alveolar resultante deste processo foi denominado QCirDental (Apêndice A) e contém 20 perguntas que devem ser respon- didas numa escala de 0 (zero) a 10 (dez), sendo que o valor zero representa nenhum incômodo durante a cirurgia, e o valor dez indica muito muito incômodo/incômodo demais/um absurdo. Portanto, quanto maior o valor obtido, maior o desconforto sentido durante o procedimento e o período perioperatório imediato. $\mathrm{O}$ impacto total pode ser medido através da soma geral dos itens ou a soma total dos itens divididos pelo numero de perguntas (Soma Total/20), sendo que, neste último caso, obtém-se o grau de impacto numa escala de $0-10$.

\section{Seleção dos pacientes e entrevista com o questionário de autopercepção de cirurgia bucal dento-alveolar}

Os pacientes foram selecionados de forma consecutiva e conforme a indicação de extração 
dentária estabelecida nos critérios vigentes, ou seja, indicação por irrecuperabilidade do dente por razões de cárie ou doença periodontal, por indicação protética, por indicação ortodôntica ou por associação a patologias. Os pacientes foram entrevistados no período pós-operatório imediato, com o objetivo de conhecer os desconfortos do procedimento cirúrgico, quando utilizou-se o instrumento proposto. As entrevistas foram conduzidas por estudantes de odontologia conhecedores do projeto de pesquisa e previamente orientados para tal atividade, além de supervisão direta de professores envolvidos no mesmo. Nesse momento fez-se necessário que se o cirurgião e seu auxiliar se ausentassem do local da entrevista, dando oportunidade para que o paciente respondesse ao entrevistador com maior sinceridade sobre a sua percepção da cirurgia.

\section{Critérios de inclusão}

Foram incluídos pacientes com indicações de extrações dentárias de rotina odontológica e cirurgias de dentes terceiros molares não impactados ou inclusos com raízes curtas e convergentes.

\section{Critérios de exclusão}

Foram excluídos pacientes menores de 18 anos e aqueles submetidos a cirurgias de dentes terceiros molares inclusos ou com qualquer grau de impacção óssea.

\section{Teste do Instrumento}

O estudo teste foi conduzido durante o período letivo do ano de 2014 e incluiu todos os pacientes atendidos (e que aceitaram participar da pesquisa) na disciplina de Cirurgia II do Curso de Odontologia da UEPG, levando-se em consideração os critérios de inclusão e exclusão.

\section{Análise de dados}

Os dados foram tabulados em Excel para Windows $^{\circledast}$ e posteriormente analisados estatisticamente em programa IBM SPSS ${ }^{\circledast}$ conforme apropriado para cada eventual necessidade. Para avaliar o grau de consistência interna das medidas obtidas foi utilizado o Coeficiente alfa de Cronbach e, para a validade do constructo foi utilizado o teste de correlação de Spearman. Para a exclusão de itens do questionário foi utilizada a correlação item-total (coeficiente de correlação com a escala como um todo), sendo indicado para a remoção o item que apresentou correlação inferior a 0,2, além de correlações negativas entre itens $^{3,12}$. Todas as análises inferenciais levaram em consideração um nível de significância quando $\mathrm{p}$ $\leq 0,05$.

\section{Resultados}

A amostra foi composta por 123 pacientes que se submeteram a cirurgias de extração dentária de rotina odontológica, sendo que a maioria foi composta por pessoas do sexo feminino (73 sujeitos ou 59\%). A idade variou entre 18 e 71 anos (Média 39, DP 13). Nenhum paciente da amostra apresentou qualquer dificuldade em entender o QCirDental, recusou-se a responder qualquer pergunta ou ponderou sobre outro desconforto. Entretanto, avaliações positivas sobre os cirurgioes e o tratamento ocorreram ocasionalmente. As questões do QCirDental que mostraram maiores índices de desconforto ou incômodo (com escores diferentes de zero) foram: Eu me senti nervoso durante a cirurgia (impacto de 48\%); A dor que eu senti durante a anestesia (impacto de 40,7\%); A sensação de ter perdido meu(s) dente(s) (impacto de 39,8\%); Os líquidos e sangue na minha boca (impacto de 35\%); A dor que eu senti durante a cirurgia (impacto de 35\%); Eu tive medo da anestesia (impacto de 34,1\%) e A impressão que eu tive dos machucados na minha boca (impacto de 27,6\%). As questões que mostraram menores índices de impacto foram: Durante a minha cirurgia, senti a minha privacidade invadida (impacto de $4,1 \%$ ); e a falta de explicações após terminar a cirurgia (impacto de $5,7 \%)$. Para 20 pacientes (16,3\% da amostra) o procedimento cirúrgico de extração dentária e a perda do(s) dente(s) não representaram nenhum desconforto ou incomodo. Todas as médias de impactos, consistência interna e responsividade podem der observados na Tabela 1.

O instrumento mostrou Coeficiente alfa de Cronbach de 0,83 e sem melhora substancial com exclusão de itens, mesmo com três questões apresentando correlação item-total inferior a 0,3, porém com valores superiores a 0,2 (Tabela 1). Dessa forma, e sendo de interesse dos pesquisadores, optou-se em manter as perguntas no instrumento. A Tabela 2 apresenta a matriz de correlação de Spearman item-item, a qual indica que as questões relacionadas à dor durante a anestesia e à sensação de ter perdido o dente apresentaram maior número de itens não correlacionados às demais questões. Observa-se, também, que todas 
Tabela 1. Itens do questionário com respectivas médias e medidas de desconforto ou incomodo (em \%) para cirurgias bucais com escores diferentes de zero além da avaliação da consistência interna através da correlação item-total e alpha de Cronbach.

\begin{tabular}{|c|c|c|c|c|c|}
\hline & $\begin{array}{l}\text { Média } \\
(0-10)\end{array}$ & $\begin{array}{l}\text { Desvio } \\
\text { Padrão }\end{array}$ & $\begin{array}{l}\text { Desconforto } \\
(\%)\end{array}$ & $\begin{array}{l}\text { Correlação } \\
\text { Item-Total }\end{array}$ & $\begin{array}{l}\text { Alpha de } \\
\text { Cronbach } \\
\text { (se item } \\
\text { removido) }\end{array}$ \\
\hline 1. Eu me senti nervoso durante a cirurgia & 1,89 & 2,8 & 48,0 &, 52 & ,82 \\
\hline $\begin{array}{l}\text { 2. Os comentários que os cirurgiões ou auxiliares } \\
\text { fizeram durante minha cirurgia }\end{array}$ &, 40 & 1,0 & 15,4 &, 56 & 82 \\
\hline 3. Os líquidos e sangue da minha boca & 1,02 & 1,9 & 35,0 & ,37 & ,82 \\
\hline $\begin{array}{l}\text { 4. A impressão que eu tive dos machucados na } \\
\text { minha boca }\end{array}$ &, 88 & 1,9 & 27,6 &, 55 &, 81 \\
\hline 5. Eu tive medo da anestesia & 1,47 & 2,7 & 34,1 & ,47 & ,82 \\
\hline 6. A dor que eu senti durante a anestesia & 1,20 & 1,9 & 40,7 &, 50 & ,82 \\
\hline 7. A dor que eu senti durante a cirurgia & 1,15 & 2,3 & 35,0 & ,43 & ,82 \\
\hline 8. Os barulhos dos instrumentos & ,78 & 1,7 & 25,2 & ,38 & ,82 \\
\hline 9. O tempo que a cirurgia levou & ,60 & 1,4 & 22,0 &, 54 &, 82 \\
\hline $\begin{array}{l}\text { 10. A falta de explicação do que estava } \\
\text { acontecendo durante a cirurgia }\end{array}$ &, 36 & 1,3 & 12,2 & ,45 &, 82 \\
\hline $\begin{array}{l}\text { 11. A falta de delicadeza ou cuidado do cirurgião } \\
\text { para comigo durante a cirurgia }\end{array}$ &, 18 &, 8 & 8,9 &, 29 & ,83 \\
\hline $\begin{array}{l}\text { 12. Eu me senti indignado durante a cirurgia } \\
\text { (qualquer motivo relacionado) }\end{array}$ &, 18 & ,7 & 7,3 &, 50 & ,82 \\
\hline $\begin{array}{l}\text { 13. A dificuldade do cirurgião para terminar a } \\
\text { cirurgia }\end{array}$ & ,65 & 1,7 & 22,8 & ,48 &, 82 \\
\hline $\begin{array}{l}\text { 14. Durante a minha cirurgia, senti minha } \\
\text { privacidade invadida }\end{array}$ & ,09 &, 5 & 4,1 &, 50 & ,83 \\
\hline 15. O lugar, o ambiente &, 25 & ,9 & 10,6 & 44 &, 82 \\
\hline 16. Os cheiros diferentes &, 27 & ,8 & 13,0 &, 28 & ,83 \\
\hline $\begin{array}{l}\text { 17. Os materiais ou instrumentos que colocaram } \\
\text { na minha boca }\end{array}$ &, 50 & 1,3 & 17,9 & ,45 & ,82 \\
\hline 18. Eu me senti angustiado durante a cirurgia & ,91 & 2,0 & 26,8 & ,63 &, 81 \\
\hline 19. A falta de explicações após terminar a cirurgia & ,13 & ,6 & 5,7 & ,32 & ,83 \\
\hline 20. A sensação de ter perdido meu(s) dente(s) & 1,99 & 3,1 & 39,8 &, 27 & ,84 \\
\hline Total & 14,8 & 17,4 & 83,7 & - & - \\
\hline Total/20 & 0,7 & 0,8 & 83,7 & - & - \\
\hline
\end{tabular}

as questões mostraram correlação positiva, com rho superior a 0,3 para todos os itens, quando comparadas à soma total dos itens/20 (Escores Totais divididos por 20). Não houveram correlações negativas.

Tanto a validade de face quanto a de conteúdo foram consideradas como satisfatórias devido à simplicidade da formulação dos itens e à facilidade cognitiva de interpretação das questões, tendo estas sido compreendidas perfeitamente pelos participantes.

A análise de componentes principais (Kaiser-Meyer-Olkin Measure of Sampling Adequacy 0,72 e Bartlett's Test of Sphericity com p $<0,001$ ) mostrou seis (6) dimensões do instrumento que explicam $67,5 \%$ da variância. Estes resultados podem ser observados na Tabela 3 e Figura 1.

Dentre as variáveis estudadas não houveram diferenças estatisticamente significantes quando os escores totais (Escores Totais divididos por 20) do QCirDental foram comparados com sexo, a presença de doença sistêmica, hábitos como o uso de álcool e tabaco, quantidade de dentes extraídos, entre outras, e nem foram observadas correlações significantes com idade e tempo cirúrgico. As variáveis que mostraram diferenças estatisticamente significantes foram a realização de retalho cirúrgico (Teste de Mann-Whitney $\mathrm{p}$ 
Tabela 2. Validade do constructo avaliada pelo teste correlação de Spearman Item-Item e Item-Total/20 com indicações de nível de significância.

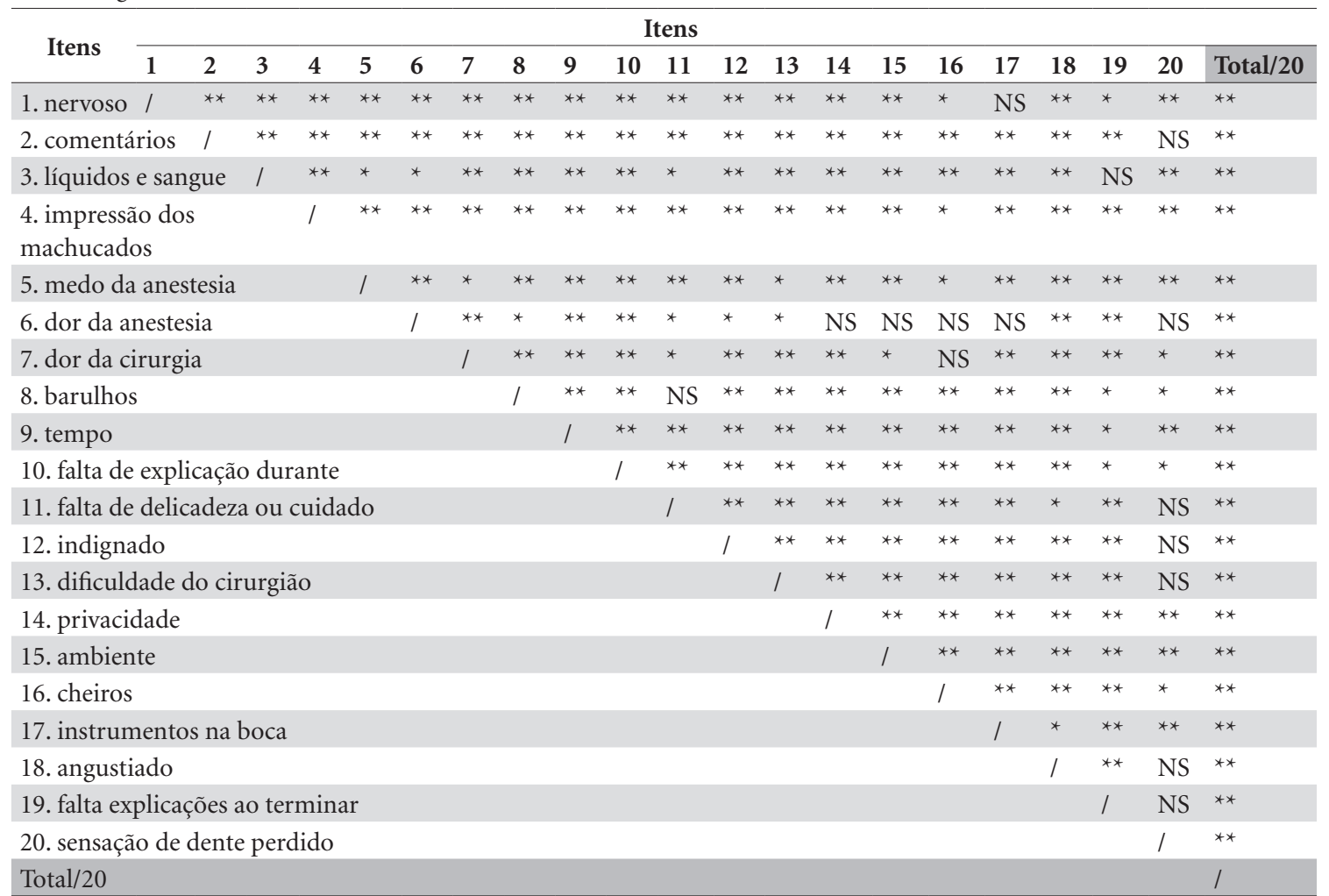

** correlação significante a um nível de significância menor que 0,01 (2-tailed). * correlação significante a um nível de significância menor que 0,05 (2-tailed). NS Não significante.

Tabela 3. Análise de componentes principais, com método de rotação Direct Oblimin com Normalização de Kaiser, sem restrições ao número de componentes, indicando seis (6) dimensões do instrumento.

\begin{tabular}{llr}
\hline \multicolumn{1}{c}{ Dimensões } & \multicolumn{1}{c}{ Itens ou questões incluidas } \\
\hline 1 & 4. A impressão que eu tive dos machucados na minha boca &, 819 \\
Eigenvalue & 12. Eu me senti indignado durante a cirurgia (qualquer motivo relacionado) &, 748 \\
5,97 & 9. O tempo que a cirurgia levou &, 693 \\
& 13. A dificuldade do cirurgião para terminar a cirurgia &, 637 \\
& 2. Os comentários que os cirurgiões ou auxiliares fizeram durante minha cirurgia &, 528 \\
& 8. Os barulhos dos instrumentos &, 344 \\
2 & 16. Os cheiros diferentes &,- 747 \\
Eigenvalue & 17. Os materiais ou instrumentos que colocaram na minha boca \\
1,99 & 14. Durante a minha cirurgia, senti minha privacidade invadida &,- 731 \\
3 & 11. A falta de delicadeza ou cuidado do cirurgião para comigo durante a cirurgia &,- 643 \\
Eigenvalue & 10. A falta de explicação do que estava acontecendo durante a cirurgia &, 874 \\
1,71 & 5. Eu tive medo da anestesia &, 779 \\
& 1. Eu me senti nervoso durante a cirurgia &, 593 \\
& 6. A dor que eu senti durante a anestesia &, 567 \\
4 & 19. A falta de explicações após terminar a cirurgia &, 473 \\
Eigenvalue & 15. O lugar, o ambiente &, 898 \\
1,42 & 18. Eu me senti angustiado durante a cirurgia &, 713 \\
5 & 3. Os líquidos e sangue da minha boca &, 410 \\
Eigenvalue & 7.A dor que eu senti durante a cirurgia &, 852 \\
1.38 & 20. A sensação de ter perdido meu(s) dente(s) &, 676 \\
6 & &, 879 \\
1,02 & &
\end{tabular}




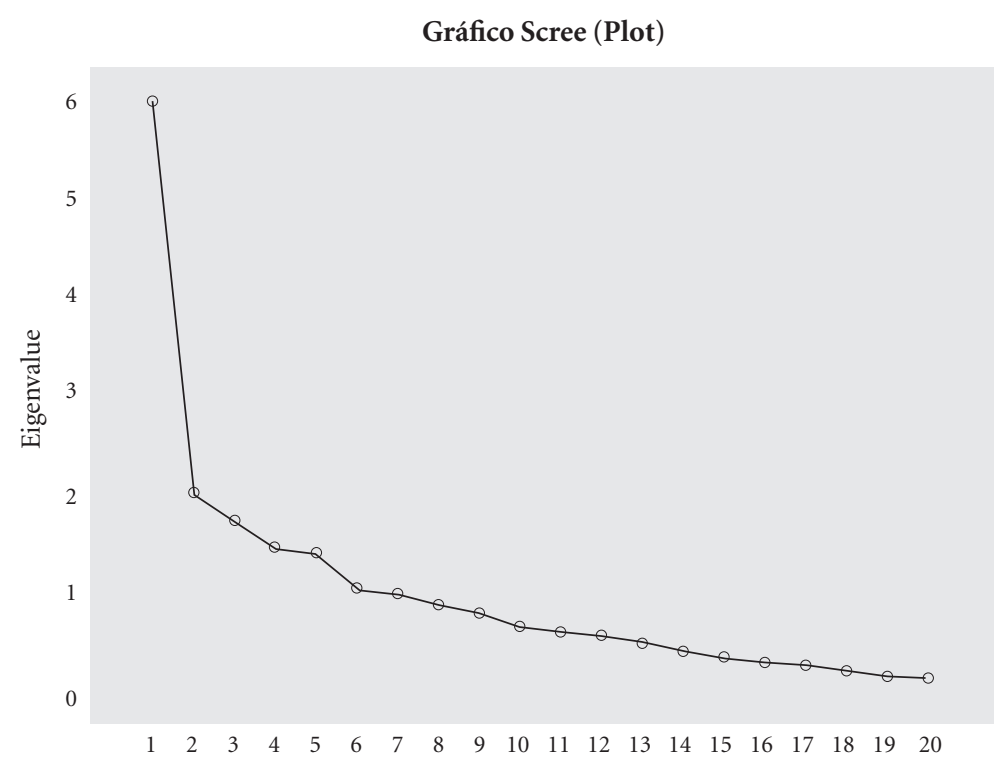

Número de componentes

Figura 1. Gráfico Scree (Scree Plot) obtido através de análise de componentes principais, com método de rotação Direct Oblimin e técnica não restritiva, indicando 6 dimensões.

$=0,009)$ e o procedimento cirúrgico haver necessitado de repetidas complementações anestésicas (Teste de Kruskal Wallis $\mathrm{p}=0,02$ ), sendo que ambas produziram impacto negativo maior. Todavia, quando o cirurgião relatou um procedimento que apresentou dificuldade técnica maior, houve impacto sobre os itens: (a) sentir-se nervoso (Teste de Mann-Whitney $\mathrm{p}=0,02)$ e (b) incomodo em relação à dificuldade do cirurgião para terminar o procedimento (Teste de Mann -Whitney $\mathrm{p}=0,03)$. Conforme o item específico, o tempo de duração do procedimento mostrou uma correlação negativa conforme a idade (teste de Correlação de Spearman rho -0,25, p = 0,004), indicando que pacientes jovens tendem a tolerar menos os procedimentos mais longos.

\section{Discussão}

A validade de um instrumento pode ser entendida como o quanto os resultados dos valores de medida possuem significado ou são interpretáveis, ou ainda, o quanto as medidas de um teste realmente medem o que se propõe a mensurar. Esse conceito amplo de validade ultrapassa a ideia de "concordância com critério" ${ }^{13}$ e se sobrepõe à fundamentação epidemiológica de sensibilidade e especificidade de um instrumento. $\mathrm{O}$ instrumento de avaliação e quantificação do impacto e percepção da cirurgia bucal (QCirDental), que buscou avaliar os sentimentos e desconfortos do paciente, mostrou-se de fácil interpretação, uma vez que se baseou em perguntas que englobaram uma representatividade de assuntos no tema da sua definição conceitual, com respostas ponderadas, facilitando as indicações em relação aos desconfortos sentidos. O QCirDental traz, portanto, a capacidade de mensurar e identificar de forma compreensível um conjunto amplo, porém convergente, de fatores relacionados ao desconforto em procedimentos cirúrgicos odontológicos, no que se refere aos estágios iniciais do desenvolvimento de um instrumento ou seja, a validade de face e conteúdo.

O questionário abre a possibilidade de melhor interpretar os incômodos, as percepções e as sensações do paciente durante o procedimento cirúrgico bucal traduzindo-se numa alternativa de avaliação viável à qualidade dos cuidados e serviços oferecidos. A introdução do QCirDental como ferramenta de avaliação toma importância, ainda, diante do fato de que apenas 16,3\% dos pacientes neste estudo não terem relatado 
nenhum desconforto, nenhum impacto, o que, visto por outro prisma, aponta para uma maioria de indivíduos, outrem ignorados quanto às suas queixas, agora sendo percebidos com a perspectiva e o objetivo de criar conforto e reduzir o estresse emocional.

Visando à qualidade de cuidados, deve também ser ressaltado que o presente estudo foi desenvolvido em um ambiente acadêmico, ou seja, no ambiente de formação dos futuros cirurgiõesdentistas, os quais tendem a incorporar melhor as tendências positivas mas também de perpetuar os vícios da profissão. Enfatizar a qualidade do atendimento pode modificar a perspectiva frequentemente observada da visão do acadêmico (ou mesmo o profissional já formado), que tende a visualizar a técnica cirúrgica como o primeiro objetivo e a remoção do dente como o prêmio e percebido como troféu. Mas como explicar o fato de que aproximadamente $40 \%$ dos pacientes reclamam da "sensação de ter (seu) meu dente perdido?" Troféus para uns, lamento para outros, todavia, não se trata de uma competição esportiva mas sim do terapeuta, da terapia e do tratado, e o enfoque da redução do dano e de desconfortos ao paciente deve ser primada.

A utilização de um instrumento validado pode permitir a comparação de qualidade de atendimento em diferentes ambientes (públicos versus privados, generalistas versus especialistas), comparar diferentes regiões e culturas, além de permitir a coleta de informações do processo relacionado à qualidade do cuidado em cirurgia bucal, levando a um melhor entendimento da percepção do paciente enquanto submetido ao procedimento cirúrgico. Reissmann et al. ${ }^{11}$ recentemente apresentaram um instrumento de medida de impacto para o período transcirúrgico e declaram que a ferramenta por eles desenvolvida é válida e confiável para avaliar a qualidade de cuidados em cirurgia bucal. Este foi o único trabalho científico identificado com o intuito específico de avaliar e de validar um instrumento desse tipo, até o desenvolvimento do presente estudo. O questionário ora apresentado mostra-se diferente do desenvolvido por Reissmann et al. ${ }^{11}$, uma vez que esse avalia o período perioperatório imediato e inclui principalmente as sensações desagradáveis e os desconfortos relacionados ao procedimento cirúrgico, não considerando, entretanto, sentimentos importantes como o estado de tensão, de angústia, percepções quanto à privacidade e o ambiente, além dos sentimentos relacionados à perda do dente, dimensões tais que estão incluídas no QCirDental. Como não levar em consideração o indicativo de que $48 \%$ dos pacientes deste estudo sentiram-se nervosos o suficiente para relatar esta sensação como incomodo relacionado ao procedimento cirúrgico?

Os critérios apresentados no presente estudo indicam que os objetivos da validação de face e conteúdo foram atingidos sendo que o instrumento apresentou ainda excelente validade interna. Entretanto, devido à ausência de instrumento validado para a língua portuguesa com o mesmo enfoque, não foi possível realizar a análise comparativa e desenvolver a validade de critério. Diante do exposto, uma segunda etapa de análise do instrumento, utilizando-se medidas repetidas, deve ser realizada a fim de verificar a confiabilidade do mesmo.

O processo de validação de medidas geralmente envolve uma série de passos conhecidos como validação de constructo ${ }^{13}$ que não aferem apenas a estrutura interna do instrumento mas o modo como ele se relaciona com outros constructos tais como qualidade de vida ou dor. Desse modo, uma terceira etapa com o QCirDental deve ser realizada com amostra maior para avaliar a relação entre diferentes constructos, além de garantir uma melhor formatação das dimensões do próprio instrumento, uma vez que a análise de componentes principais demanda amostras com um número superior a 300 sujeitos. Amostras maiores tendem a estabilizar o padrão do componente, a minimizar a probabilidade de erros, a maximizar a acurácia das estimativas da população, além de permitir aumentar a generalização dos resultados. Por outro lado, há autores que admitem amostras entre 100 e 200 indivíduos, da mesma forma que consideram que, submetida ao teste de Kaiser-Meyer-Olkin, uma amostra é adequada quando apresenta resultado superior a $0,7^{1,3}$, o que também foi observado no presente estudo $(0,72)$.

Por fim, no processo de validação, também deverão ser incluídos procedimentos cirúrgicos bucais de maior complexidade como as de remoção de terceiros molares inclusos, as remoções dentárias amplas associadas às alveoplastias, as cirurgias com acessos maiores tanto associadas às remoções dentárias, bem como aquelas onde a remoção dentária está associada a processos patológicos do tecido ósseo, oportunizando, dessa forma, uma melhor caracterização da especificidade e sensibilidade do instrumento. 


\section{Conclusões}

Neste estudo foi possível determinar que o instrumento ou o questionário de autopercepção de cirurgia bucal dento-alveolar (QCirDental) possui excelente consistência interna, sendo um instrumento de fácil leitura e interpretação, compatível com uma adequada validade semântica e de conteúdo. Mais de $80 \%$ dos pacientes submetidos às extrações dentárias de rotina odontológica apresentaram algum desconforto ou incomodo no período transoperatório o que ressalta a necessidade de utilização de um instrumento de medida de avaliação da qualidade de cuidados, procedimentos ou serviços, bem como, de buscar formas de minimizar os impactos negativos.

\section{Colaboradores}

MC Bortoluzzi foi o idealizador do projeto, coordenação, coleta de dados, análise de dados, redação do manuscrito. L Martins e A Takahashi participaram da coleta de dados, comitê de especialista, revisão geral. B Ribeiro contribuiu na coleta e na tabulação de dados. L Martins participou da tabulação de dados, revisão da estatística e revisão crítica. MH Baldani Pinto foi idealizadora do projeto, e contribuiu na revisão crítica. 


\section{Referências}

1. Johnson J, Carlsson S, Johansson M, Pauli N, Rydén A, Fagerberg-Mohlin B, Finizia C. Development and validation of the Gothenburg Trismus Questionnaire (GTQ). Oral Oncol 2012; 48(8):730-736.

2. Ni Riordain R, Meaney S, McCreary C. A patient-centered approach to developing a quality-of-life questionnaire for chronic oral mucosal diseases. Oral Surg Oral Med Oral Pathol Oral Radiol Endod 2011; 111(5):578-586.

3. Montero J, Bravo M, López-Valverde A. Development of a specific indicator of the well-being of wearers of removable dentures. Community Dent Oral Epidemiol 2011; 39(6):515-524.

4. Beetz I, Burlage FR, Bijl HP, Hoegen-Chouvalova O, Christianen ME, Vissink A, van der Laan BF, de Bock GH, Langendijk JA. The Groningen Radiotherapy-Induced Xerostomia questionnaire: development and validation of a new questionnaire. Radiother Oncol 2010;97(1):127-131.

5. Ohrbach R, Granger C, List T, Dworkin S. Preliminary development and validation of the Jaw Functional Limitation Scale. Community Dent Oral Epidemiol 2008; 36(3):228-236.

6. Pau A, Croucher R, Marcenes W, Leung T. Development and validation of a dental pain-screening questionnaire. Pain 2005; 119(1-3):75-81.

7. Aggarwal VR, Lunt M, Zakrzewska JM, Macfarlane GJ, Macfarlane TV. Development and validation of the Manchester orofacial pain disability scale. Community Dent Oral Epidemiol 2005; 33(2):141-149.

8. Cunningham SJ, Garratt AM, Hunt NP. Development of a condition-specific quality of life measure for patients with dentofacial deformity: I. Reliability of the instrument. Community Dent Oral Epidemiol 2000; 28(3):195-201.
9. Kämmerer PW, Palarie V, Daubländer M, Bicer C, Shabazfar N, Brüllmann D, Al-Nawas B. Comparison of $4 \%$ articaine with epinephrine $(1: 100,000)$ and without epinephrine in inferior alveolar block for tooth extraction: double-blind randomized clinical trial of anesthetic efficacy. Oral Surg Oral Med Oral Pathol Oral Radiol 2012; 113(4):495-499.

10. Bortoluzzi MC, Traebert J, Lasta R, Da Rosa TN, Capella DL, Presta AA. Tooth loss, chewing ability and quality of life. Contemp Clin Dent 2012; 3(4):393-397.

11. Reissmann DR, Semmusch J, Farhan D, Smeets R, Heiland M, Heydecke G. Development and validation of the Burdens in Oral Surgery Questionnaire (BiOS-Q). J Oral Rehabil 2013; 40(10):780-787.

12. Goes PSA, Fernandes LMDA, Lucena LBS. Validação de Instrumentos de Coleta de Dados. In: Antunes JLF, Peres MA, organizadores. Fundamentos de Odontologia - Epidemiologia da Saúde Bucal. Rio de Janeiro: Guanabara Koogan; 2006. p. 390-397.

13. McDowell, I. Measuring health: a guide to rating scales and questionnaires. $3^{\text {rd }}$ ed. Oxford: Oxford University Press; 2006

Artigo apresentado em 08/06/2015

Aprovado em 17/12/2015

Versão final apresentada em 19/12/2015 